\title{
An Automatic Identification Authentic Work Anti-counterfeiting Algorithm Based on DWT-DCT
}

\author{
Yue Zhang and Jingbing $\mathrm{Li}^{*}$ \\ College of Information Science and Technology, \\ Hainan University, Haikou, 570228, China \\ artistyue@163.com,jingbingli2008@hotmail.com
}

\begin{abstract}
In order to restrain the bad influences of fake and inferior products, an automatic identification authentic work anti-counterfeiting algorithm is proposed in this paper. The authentic work anti-counterfeiting uses a unique random character structure to carry out security. The algorithm is convenient for customers to distinguish the anti-counterfeiting tag by both naked eye and mobile. There is not yet a similar algorithm published. In the algorithm, the feature vectors are extracted using DWT-DCT. Using extracted feature vectors from anti-counterfeiting tag to realize automatic identification. Therefore, this algorithm saves the storage space and improves the identification rate. In addition, the experimental results show this algorithm have strong robustness under common attacks, geometrical attacks.
\end{abstract}

Keywords: Anti-counterfeiting; Automatic identification; Feature vector; DWT-DCT; Robustness

\section{Introduction}

Anti-counterfeit got quick development in a short span of twenty years [1], because the number of proliferation of fake and inferior increase swift. Meanwhile, they try different anti-counterfeiting technologies to reduce the loss caused by fake products [2]. Traditional external packaging methods have many drawbacks, such as reproducibility, short period, so that restricted technology application [3-4]. In previous years, bar code, two-dimensional code are popular anti-counterfeiting technologies. These technologies can realize automatic identification, but have the shortcomings of being easily forged, and being difficult to be recognized by consumers [5-7]. Recent anti-counterfeiting research hotspot is RFID [8-10]. RFID is very good but expensive. At the same time, there are some anti-counterfeiting technologies combine new technologies such as biology, chemistry [11]. However, these anti-counterfeiting technologies need special identification conditions. This paper presents an automatic identification authentic work anti-counterfeiting algorithm. Each security label has a unique text pattern that is randomly carved, so it can prevent the labels from being copied. We establish security database of feature vectors based on DWT-DCT coefficient signs. This algorithm can be easy identification of consumers and mobile, and has good robustness.

\section{The fundamental Theory}

\subsection{The Discrete Wavelet Transform (DWT)}

Wavelet transforms, which proposed by Daubechies and Mallat, are generated from simple orthogonal transforms. Wavelet transform can be used for various applications of

* Corresponding Author 
image processing [12]. Define the wavelet function $\Psi_{a, b}(t)$ as the base, and the wavelet transform of $f \in L^{2}(R)$ by $\Psi$ is defined as:

$W_{f(a, b)}=\int_{R} f(t) \bar{\psi}_{a, b}(t) d t$

where, the wavelet function $\Psi_{a, b}(t)$ is obtained by translating and scaling the same base $\Psi$.

$\psi_{a, b}(\mathrm{t})=|a|^{-1 / 2} \psi((t-b) / a) \quad a, b \in R, a \neq 0$

Where, $\Psi$ is called the base wavelet, a is the dilation factor, $\mathrm{b}$ is the translation factor.

The decomposing equation of the Mallat algorithm is as follows:

$c_{j+1, k}=\sum_{n \in z} c_{j, n} \bar{h}_{n-2 k} \quad k \in z$

$d_{j+1, k}=\sum_{n \in Z} c_{j, n} \bar{g}_{n-2 k} \quad k \in Z$

The reconstruction equation of the Mallat algorithm is given by:

After the decomposing, we got four sub images (One is the low frequency approximation sub image, and the other three are the high frequency detail sub images.).

\subsection{The Discrete Cosine Transform (DCT)}

Discrete cosine transform (DCT) is the orthogonal transformation method proposed by N.Ahmed et. al., in 1974 [13]. DCT transform uses the nature of the Fourier transform transforming the image into even function form, and then doing 2-D Fourier transform so that get results with cosine form. All DCT multiplications are on real numbers. When applied to an $\mathrm{M} \times \mathrm{N}$ size image or matrix, the 2D-Discrete Cosine Transform will compresses all the energy information of the image and concentrates it in a few coefficients located in the upper left corner of the resulting real-valued $\mathrm{M} \times \mathrm{N}$ DCT/frequency matrix [14]. 2-D discrete cosine transform is defined as:

$F(u, v)=\mathrm{c}(\mathrm{u}) \mathrm{c}(\mathrm{v}) \sum_{x=0}^{M-1} \sum_{y=0}^{N-1} f(x, y) \cos \frac{\pi(2 x+1) \mu}{2 M} \cos \frac{\pi(2 y+1) v}{2 N} \quad v=0,1, \cdots, N-1 ; u=0,1, \cdots, M-1 ;$

where $\mathrm{M} \times \mathrm{N}$ is anti-counterfeiting image size, $f(x, y)$ corresponds to the value of the anti-counterfeiting image at the point $(x, y)$ and $F(u, v)$ is the DCT coefficient at the point $(u, v)$ in frequency domain. The Formula shows that the sign of DCT coefficients are related to the phase of the component.

\subsection{Authentic Work Anti-Counterfeiting}

Authentic work anti-counterfeiting technology scheme used in this paper is defined as follow. First, select words or phrase. Secondly, arbitrary rotate every words. Then sculpture these words on commodity packaging at random. Finally, we get a unique random character structure to carry out security. 


\subsection{A Method to Obtain the Feature Vector of Authentic Work Anti-Counterfeiting Image}

Choose an image to handle by DWT. Then we obtain four sub images. The high frequency detail sub images contain the edge information of the image, so that it is vulnerable to external interference. However, the low frequency approximation sub image contains the basic information of the image and has little influenced of external interference. So we handle the low frequency approximation sub image by DCT. Then we choose 8 low-frequency DCT coefficients $(\mathrm{C} 1, \mathrm{C} 2, \ldots, \mathrm{C} 8)$, which are shown in Table 1. We can find the sign of DCT coefficients are almost unchanged even after attacks, shown as Table 1 . Therefore, we let "1" represents a positive or zero coefficient, and " 0 " represents a negative coefficient so that we get a sign sequence of low-frequency DCT coefficients as feature vector of authentic work anti-counterfeiting image, as shown in column "L11" in Table 1. Finally, we compute the value of the normalized crosscorrelation (NC) which used 32 bits sign sequence low-frequency DCT coefficients to obtain accurate calculation results. After kinds of attacks, the value of $\mathrm{NC}$ is " 1.0 " or near "1.0", as shown in column "L12" in Table 1. Consequently, the sequence of DCT coefficient signs can be used as the feature vector of authentic work anti-counterfeiting image.

In order to further prove this conclusion, we selected 10 authentic work anticounterfeiting images which shown as Figure 1, to extract the feature vector and compared the correlation coefficients. And from Table 2, we can find the correlation coefficients of different images are very small. In addition to the correlation coefficient between V5 and V8 is relatively large. That's reason is V5 and V8 are almost the same image.

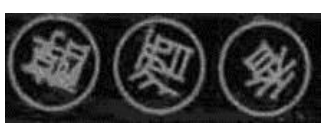

V1

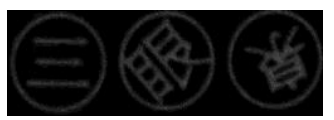

V5

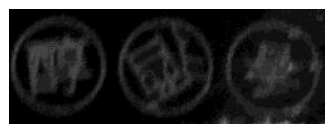

V9

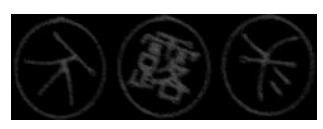

V2

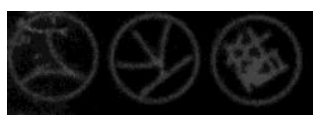

V6

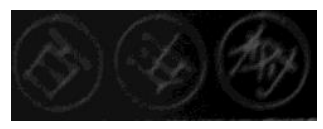

V10

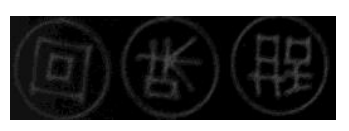

V3

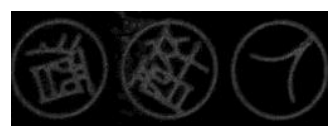

V7

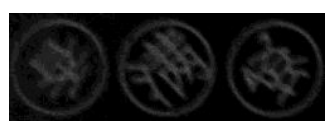

V4

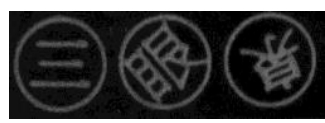

V8

Figure 1. 10 Different Authentic Work Anti-Counterfeiting Images

\section{The Algorithm}

We choose an authentic work anti-counterfeiting image as the original authentic work anti-counterfeiting image. It is described as: $F=\{f(i, j) \mid f(i, j) \in R ; 1 \leq i \leq N 1,1 \leq j \leq N 2\}$

Where $f(i, j)$ means the pixel gray value of the original texture image.

\subsection{Establish Security Database of Authentic Work Anti-Counterfeiting Image Feature Vectors}

Step 1: Acquire the feature vectors of the original authentic work anti-counterfeiting images using DWT-DCT 
Table 1. Change of DCT Low-Frequency Coefficients with Respect to Different Attacks

\begin{tabular}{|c|c|c|c|c|c|c|c|c|c|c|c|}
\hline L1 & L2 & L3 & L4 & L5 & L6 & L7 & L8 & L9 & L10 & L11 & L12 \\
\hline $\begin{array}{c}\text { Image } \\
\text { processing }\end{array}$ & $\begin{array}{l}\text { PSNR } \\
(\mathrm{dB})\end{array}$ & C 1 & $\mathrm{C} 2$ & C3 & C4 & C5 & C6 & C 7 & C8 & $\begin{array}{c}\text { Sequence } \\
\text { of } \\
\text { coefficient } \\
\text { signs } \\
\end{array}$ & NC \\
\hline $\begin{array}{c}\text { Original } \\
\text { image }\end{array}$ & $86.48 \mathrm{~dB}$ & 62.10 & 4.44 & 0.43 & 0.05 & -0.06 & 0.24 & -20.73 & -0.76 & $\begin{array}{l}1111 \\
0100\end{array}$ & 1.0 \\
\hline $\begin{array}{c}\text { Gaussian } \\
\text { noise }(2 \%)\end{array}$ & $14.42 \mathrm{~dB}$ & 67.88 & 4.18 & 0.97 & 0.03 & -0.19 & -0.55 & -19.11 & -0.66 & $\begin{array}{l}1111 \\
0000 \\
\end{array}$ & 0.8 \\
\hline $\begin{array}{c}\text { JPEG } \\
\text { compression } \\
(3 \%)\end{array}$ & $17.89 \mathrm{~dB}$ & 63.82 & 5.00 & 0.16 & -0.56 & -0.63 & 0.18 & -19.33 & -0.45 & $\begin{array}{l}1110 \\
0100\end{array}$ & 0.75 \\
\hline $\begin{array}{c}\text { Median filter } \\
{[3 \times 3]}\end{array}$ & $23.14 \mathrm{~dB}$ & 61.22 & 4.46 & 0.15 & 0.18 & -0.26 & 0.10 & -21.60 & -0.78 & $\begin{array}{l}1111 \\
0100 \\
\end{array}$ & 0. \\
\hline Rotation $\left(2^{\circ}\right)$ & $14.96 \mathrm{~dB}$ & 60.85 & 3.91 & 0.45 & 0.03 & -0.03 & 0.41 & -20.52 & -0.43 & $\begin{array}{l}1101 \\
0100\end{array}$ & 0.9 \\
\hline Scaling $(\times 2)$ & & 12.42 & 0.89 & 0.09 & 0.01 & -0.01 & 0.05 & -4.15 & -0.15 & $\begin{array}{l}1111 \\
0100 \\
\end{array}$ & 1.0 \\
\hline $\begin{array}{c}\text { Scaling } \\
(\times 0.5) \\
\end{array}$ & & 31.07 & 2.22 & 0.21 & 0.03 & -0.02 & 0.13 & -10.34 & -0.39 & $\begin{array}{l}1111 \\
0100 \\
\end{array}$ & 1. \\
\hline $\begin{array}{c}\text { Left } \\
\text { translation } \\
(2 \%) \\
\end{array}$ & $11.82 \mathrm{~dB}$ & 61.72 & 6.31 & 0.20 & 2.33 & -0.38 & 4.69 & -20.30 & -2.90 & $\begin{array}{l}1111 \\
0100\end{array}$ & 0. \\
\hline $\begin{array}{c}\text { Down } \\
\text { translation } \\
(2 \%) \\
\end{array}$ & $14.30 \mathrm{~dB}$ & 61.29 & 4.36 & 0.42 & -0.01 & -0.06 & 0.18 & -20.76 & -0.78 & $\begin{array}{l}1110 \\
0100\end{array}$ & 0.82 \\
\hline $\begin{array}{c}\text { Cropping } \\
(2 \% \text { from } Y)\end{array}$ & & 62.11 & 4.42 & 0.42 & -0.01 & -0.06 & 0.19 & -21.03 & -0.79 & $\begin{array}{l}1110 \\
0100 \\
\end{array}$ & 0.8 \\
\hline $\begin{array}{c}\text { Cropping } \\
(2 \% \text { fromX })\end{array}$ & & 62.39 & 3.29 & 1.53 & -1.29 & 1.67 & -2.77 & -19.63 & 0.47 & $\begin{array}{l}1110 \\
1001 \\
\end{array}$ & 0.6 \\
\hline
\end{tabular}

Unit of transform coefficient: $1.0 \mathrm{e}+002$, NC used 32 bits sign sequence low-frequency DCT coefficients to obtain

Firstly, handle the original image $\mathrm{F}(\mathrm{i}, \mathrm{j})$ by DWT to obtain the low frequency approximation sub image FA(i,j). Secondly, handle the low frequency approximation sub image by DCT to obtain the coefficient matrix $\mathrm{FD}(\mathrm{i}, \mathrm{j})$. select the previous $4 \times 8$ coefficients to compose FD32(i,j). Finally, do the symbol operation on coefficients to obtain the feature vectors $\mathrm{V}(\mathrm{j})$. The procedure is described as:

$$
\begin{aligned}
& \boldsymbol{F A}(i, j)=\mathrm{D} \mathrm{W} T 2(\boldsymbol{F}(i, j)) \\
& \boldsymbol{F} \boldsymbol{D}(i, j)=\operatorname{DCT} 2(\boldsymbol{F}(i, j)) \\
& \boldsymbol{V}(j)=\operatorname{Sign}\left(\boldsymbol{F} \boldsymbol{D}_{32}(i, j)\right)
\end{aligned}
$$

Step 2: Save the feature vectors of the original authentic work anti-counterfeiting images in database

Repeat operations as step 1, till all authentic work anti-counterfeiting images are handled. Then Save these feature vectors in security database. 
Table 2. Correlation Coefficients of Feature Vector of Different Images

\begin{tabular}{|c|c|c|c|c|c|c|c|c|c|c|}
\hline & V1 & V2 & V3 & V4 & V5 & V6 & V7 & V8 & V9 & V10 \\
\hline $\mathrm{V} 1$ & $\underline{1.00}$ & 0.154 & 0.071 & 0.189 & 0.126 & 0.288 & 0.118 & 0.076 & 0.118 & 0.126 \\
\hline V2 & 0.154 & 1.00 & 0.159 & 0.156 & 0.406 & $\begin{array}{c}- \\
0.062 \\
\end{array}$ & 0.155 & 0.248 & $\begin{array}{c}- \\
0.221 \\
\end{array}$ & 0.156 \\
\hline V3 & 0.071 & 0.159 & 1.00 & 0.125 & 0.125 & 0.029 & $\begin{array}{c}- \\
0.122\end{array}$ & 0.105 & $\begin{array}{c}- \\
0.122\end{array}$ & 0.188 \\
\hline V4 & 0.189 & 0.156 & 0.125 & 1.00 & 0.063 & $\begin{array}{c}- \\
0.031 \\
\end{array}$ & 0.125 & 0.032 & 0.313 & 0.125 \\
\hline V5 & 0.126 & 0.406 & 0.125 & 0.063 & $\underline{1.00}$ & 0.156 & 0.00 & 0.791 & 0.00 & 0.188 \\
\hline V6 & 0.288 & $\begin{array}{c}- \\
0.062 \\
\end{array}$ & 0.029 & $\begin{array}{c}- \\
0.031 \\
\end{array}$ & 0.156 & $\underline{1.00}$ & $\begin{array}{c}- \\
0.217 \\
\end{array}$ & 0.068 & 0.096 & 0.219 \\
\hline V7 & 0.118 & 0.155 & $\begin{array}{c}- \\
0.122\end{array}$ & 0.125 & 0.00 & 0.217 & 1.00 & 0.212 & 0.184 & $\begin{array}{c}- \\
0.063\end{array}$ \\
\hline V8 & 0.076 & 0.248 & 0.105 & 0.032 & 0.791 & 0.068 & 0.212 & 1.00 & $\begin{array}{c}- \\
0.042\end{array}$ & 0.158 \\
\hline V9 & 0.118 & $\begin{array}{c}- \\
0.221 \\
\end{array}$ & $\begin{array}{c}- \\
0.122 \\
\end{array}$ & 0.313 & 0.00 & 0.096 & 0.184 & $\begin{array}{c}- \\
0.042\end{array}$ & $\underline{1.00}$ & $\underline{0.125}$ \\
\hline V10 & 0.126 & 0.156 & 0.188 & 0.125 & 0.188 & 0.219 & $\begin{array}{c}- \\
0.063\end{array}$ & 0.158 & 0.125 & 1.00 \\
\hline
\end{tabular}

\subsection{The Automatic Identification of Authentic Work Anti-Counterfeiting Image}

Step 3: Acquire the feature vector of the tested image

To acquire the feature vector $V^{\prime}(j)$ of tested image $F^{\prime}(i, j)$, do operations which is similar as step 1 . The procedure is described as:

$$
\begin{aligned}
& \boldsymbol{F} \boldsymbol{A}^{\prime}(i, j)=\mathrm{DW} T 2\left(\boldsymbol{F}^{\prime}(i, j)\right) \\
& \boldsymbol{F} \boldsymbol{D}^{\prime}(i, j)=\operatorname{DCT} 2\left(\boldsymbol{F}^{\prime}(i, j)\right) \\
& \boldsymbol{V}^{\prime}(j)=\operatorname{Sign}\left(\boldsymbol{F} \boldsymbol{D}_{32}{ }^{\prime}(i, j)\right)
\end{aligned}
$$

Step 4: Calculate the Peak Signal to Noise Ratio (PSNR)

PSNR is used to assess the quality of the image. The bigger PSNR represents the better quality of the image. It is defined as:

$$
\left.P S N R=101 g \mid \frac{\left.M \max _{i, j}\left(\mathrm{I}_{(i, j)}\right)^{2}\right\rceil}{\sum_{i} \sum_{j}\left(\mathrm{I}_{(i, j)}-\mathrm{I}_{(i, j)}\right)^{2}}\right\rfloor
$$

Step 5: Calculate the normalized cross-correlation (NC) to determine whether the original image

Calculate the normalized cross-correlation (NC) using the formula as follow: 


$$
N C=\frac{\boldsymbol{V}(i) \times \boldsymbol{V}^{\prime}(j)}{\boldsymbol{V}^{2}(j)}
$$

The larger the value of $\mathrm{NC}$ is, the more approximation between the tested authentic work anti-counterfeiting image $\boldsymbol{F}^{\prime}(i, j)$ and the original authentic work anti-counterfeiting image $\boldsymbol{F}(i, j)$.

Step 6: Return the maximum value of NC to the user's phone

All steps above are shown in the Figure 2.

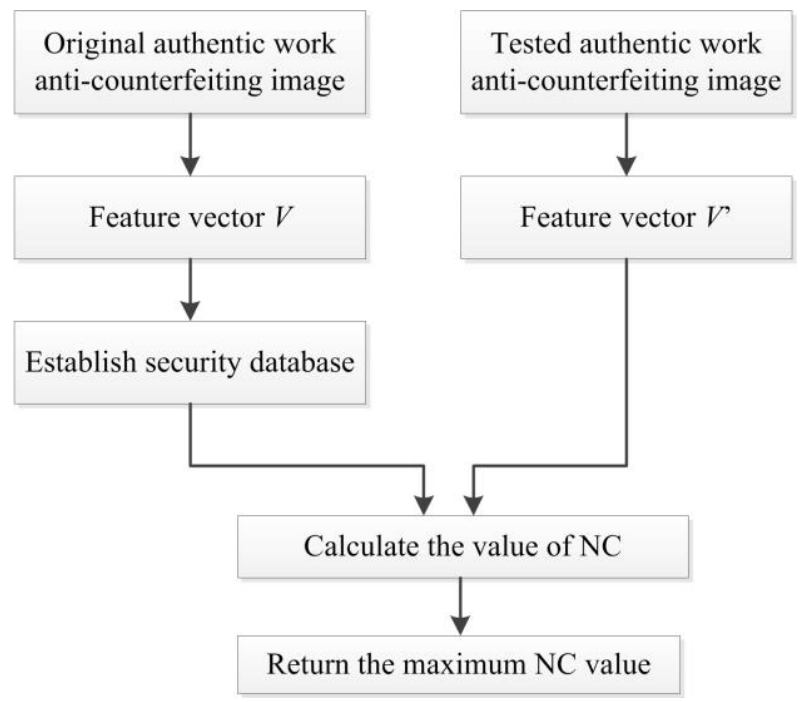

Figure 2. Authentic Work Anti-Counterfeiting Algorithm Figure

\section{Experiements}

In our experiments, we use 1000 groups of independent binary pseudo morph sequences. Every sequence consists of 32 bits. In the experiments, the 500th group is selected at random from the 1000 groups as the embedded feature vector. The size of authentic work anti-counterfeiting image is $220 \times 76$, the image is shown in Figure 3(a). The original authentic work anti-counterfeiting image is denoted as $\boldsymbol{F}(i, j)$, where $1 \leq \mathrm{i} \leq$ $220,1 \leq \mathrm{j} \leq 76$. The corresponding coefficient matrix is $\boldsymbol{F D}(i, j)$. We select the 32 bits sequence of coefficient signs as the feature vector $\boldsymbol{V}(j)$ selected from $\boldsymbol{F D}(i, j)$, where $1 \leq$ $\mathrm{i} \leq 4,1 \leq \mathrm{j} \leq 8$. Security database is comprised of 32 bits feature vectors so that database can save more space than authentic work anti-counterfeiting images. By this way, we improve the rate of automatic identification.

In this simulation, PSNR is used for objectively evaluating the quality of the tested image, and the NC is used to objectively evaluate the results of similarity detection.

Figure 3(b) is the NC values between the 1000 available pseudo morph sequences and the extracted feature vector, which is achieved by using DWT-DCT and symbolic operation. It can be seen from Figure 3(a) that the original authentic work anticounterfeiting image. The similarity can be detected clearly, $\mathrm{NC}=1.0$.

\subsection{Common Attacks}

4.1.1. Gaussian noise attacks: Table 3 is the experimental data of anti-Gauss noise interference of anti-counterfeiting image. When the Gauss noise intensity of up to $15 \%$, the PSNR of the anti-counterfeiting image down to $6.81 \mathrm{~dB}$, the image is obscure. Then extract the anti-counterfeiting image, So we can conclude that the algorithm has strong 
anti-Gauss noise ability. Figure 4(a) shows the authentic work anti-counterfeiting image under Gaussian attacks (2\%). The value PSNR is $14.35 \mathrm{~dB}$. The similarity can be detected with $\mathrm{NC}=0.94$, as shown in Figure 4(b).

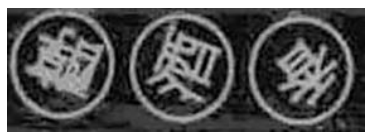

(a)

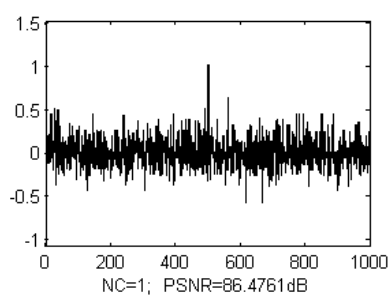

(b)

Figure 3. Original Authentic Work Anti-Counterfeiting Image and Detecting NC without Attacks: (a) Original Authentic Work Anti-Counterfeiting Image; (b) Similarity Detector

Table 3. Anti-Gauss Noise Data

\begin{tabular}{|c|c|c|c|c|c|c|}
\hline Noise Intensity (\%) & 1 & 2 & 3 & 5 & 10 & 15 \\
\hline PSNR(dB) & 17.24 & 14.42 & 12.81 & 10.78 & 8.18 & 6.81 \\
\hline NC & 0.88 & 0.82 & 0.76 & 0.76 & 0.65 & 0.68 \\
\hline
\end{tabular}

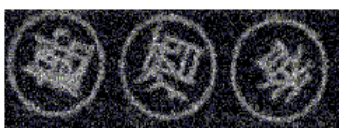

(a)

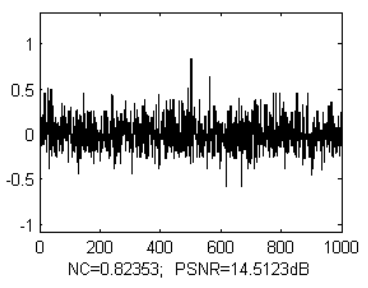

(b)

Figure 4. Gaussian Noise (noise intensity=2\%): (a) Adding Gaussian Noise; (b) Similarity Detector

4.1.2. JEPG attacks: Table 4 is anti-JPEG compression experimental data for anticounterfeiting image. When the compression quality is only $2 \%$, the image has blocking artifacts. $\mathrm{NC}=0.75$, we still can extract the anti-counterfeiting image at this situation. The results show that the algorithm is robust to JPEG attacks. The authentic work anticounterfeiting image with JPEG attacks (2\%) is shown in Figure 5(a). PSNR=17.12dB. The similarity can still be detected, $\mathrm{NC}=0.75$, as shown in Figure 5(b).

Table 4. Anti-jpeg Compression Data

\begin{tabular}{|c|c|c|c|c|c|c|}
\hline JPEG Compression (\%) & 2 & 5 & 10 & 20 & 30 & 40 \\
\hline PSNR(dB) & 17.12 & 20.60 & 24.01 & 27.24 & 29.11 & 30.33 \\
\hline NC & 0.75 & 0.75 & 0.93 & 1.0 & 1.0 & 0.82 \\
\hline
\end{tabular}

\subsection{Geometrical Attacks}

4.2.1. Rotation attacks: Table 5 is anti-rotation attacks experimental data for anticounterfeiting image. We can see when the anti-counterfeiting image clockwise rotation $10^{\circ}, \mathrm{NC}=0.69$, the anti-counterfeiting image can still be accurate to extract. Figure 6(a) shows the authentic work anti-counterfeiting image rotated clockwise by $3^{\circ}$, PSNR 
$=12.97 \mathrm{~dB}$. Figure $6(\mathrm{~b})$ shows that the similarity can be detected with $\mathrm{NC}=0.75$. Therefore we can conclude that our scheme is robust against rotation attacks.

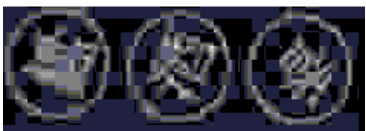

(a)

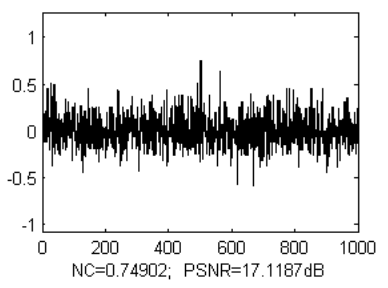

(b)

Figure 5. JPEG Compression (2\%): (a) Image After JPEG Compression; (b) Similarity Detector.

Table 5. Anti-Rotation Attacks Data

\begin{tabular}{|c|c|c|c|c|c|c|c|c|c|}
\hline $\begin{array}{c}\text { Rotational } \\
\text { Degree }\end{array}$ & $0^{\circ}$ & $1^{\circ}$ & $2^{\circ}$ & $3^{\circ}$ & $4^{\circ}$ & $5^{\circ}$ & $6^{\circ}$ & $7^{\circ}$ & $10^{\circ}$ \\
\hline PSNR(dB) & 86.48 & 19.67 & 14.96 & 12.97 & 12.05 & 11.60 & 11.31 & 11.06 & 10.51 \\
\hline $\mathrm{NC}$ & 1.0 & 1.0 & 0.94 & 0.75 & 0.67 & 0.55 & 0.68 & 0.76 & 0.69 \\
\hline
\end{tabular}

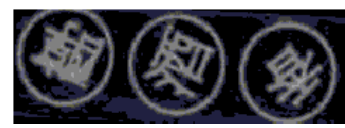

(a)

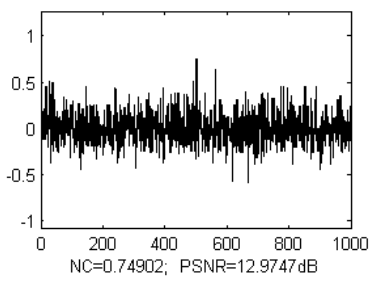

(b)

Figure 6. Rotation Attacks $\left(3^{\circ}\right)$ : (a) Under Rotation Attack; (b) Watermarking Detector

4.2.2. Scaling attacks : Table 6 is anti-scaling attacks experimental data for anticounterfeiting image. When the anti-counterfeiting image scaling factors low to 0.2 , $\mathrm{NC}=0.94$, we can extract the anti-counterfeiting image. When the anti-counterfeiting image scaling factors up to $2.0, \mathrm{NC}=1.0$, we also can extract the anti-counterfeiting image. Figure 7(a) shows the authentic work anti-counterfeiting image shrunk with a scale factor of 0.5. Figure 7(b) shows that the similarity can be detected with $\mathrm{NC}=1.0$. Hence, our algorithm is robust to scale attacks.

Table 6. Anti-Scaling Attacks Data

\begin{tabular}{|c|c|c|c|c|c|c|c|c|}
\hline Scaling Factors & 0.2 & 0.5 & 0.6 & 0.7 & 0.8 & 1.1 & 1.5 & 2.0 \\
\hline NC & 0.88 & 1.0 & 1.0 & 1.0 & 1.0 & 0.94 & 1.0 & 1.0 \\
\hline
\end{tabular}

4.2.3. Translation attacks : Table 7 is anti-left translation attacks experimental data for anti-counterfeiting image. When the anti-counterfeiting image left translation 6\%, $\mathrm{NC}=0.57$, the anti-counterfeiting image can be accurate to extract. When the anticounterfeiting image down translation $6 \%, \mathrm{NC}=0.57$. Therefore we can conclude that our scheme is robust against translation attacks. Figure 8(a) shows the authentic work anticounterfeiting image after left translation $4 \%$. Figure 8 (b) shows that the similarity can be detected with $\mathrm{NC}=0.69$. 


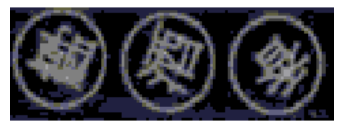

(a)

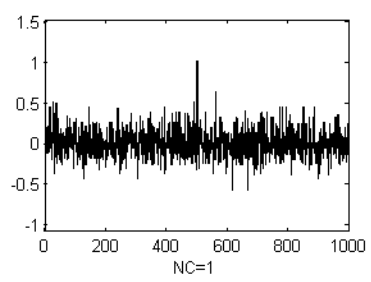

(b)

Figure 7. Scaling Attacks (×0.5): (a) Under Scaling Attack; (b) Similarity Detector

Table 7. Anti-Translation Attacks Data

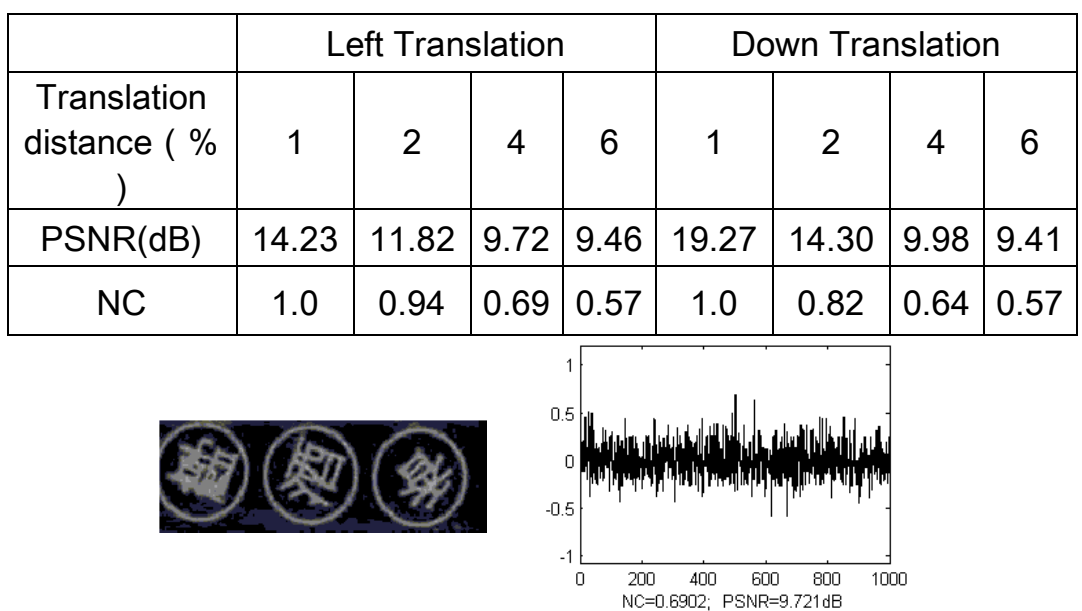

(a)

(b)

Figure 8. Left Translation Attacks (4\%): (a) Under Translation Attack; (b) Similarity Detector

\section{Conclusion}

An automatic identification algorithm of the authentic work anti-counterfeiting labels based on DWT-DCT is proposed in this paper. Authentic work anti-counterfeiting is a novel anti-counterfeiting algorithm. It is convenient for consumers to identify with both naked eyes and automatic identify. The experimental results show this algorithm can realize automatic identification and have strong robustness by combining DWT, DCT, feature vector, and data base. In addition, the algorithm can improve the rate of identification and save a lot of storage space.

\section{References}

[1] S. Sheng and X. Wu, "A new digital anti-counterfeiting scheme based on chaotic cryptography", ICT Convergence (ICTC)., Jeju Island, (2012), pp. 687-691.

[2] Y. Guan, J. Hu and Y. Li, "A new anti-counterfeiting method: fluorescent labeling by safranine $\mathrm{T}$ in tobacco seed", Acta Physiol Plant, vol. 33, (2011), pp. 1271-1276.

[3] X. Tie and J. Zou, "A Novel Digital Watermarking Method for Commercial Bills Based on V Systems", Pervasive Computing and Applications (ICPCA), (2007), pp. 389-393.

[4] Y. Tian, Z. Li and F. He, "A novel anti-counterfeiting method: Application and decomposition of RB for broad bean seeds (Vicia faba L.)", Industrial Crops and Products, vol. 61, (2014), pp. 278-283.

[5] S. Liu, "Anti-counterfeit system based on mobile phone QR code and fingerprint", Intelligent HumanMachine Systems and Cybernetics (IHMSC)., (2010), pp. 236-240. 
[6] A. Sun, Y. Sun and C. Liu, "The QR-code reorganization in illegible snapshots taken by mobile phones", Fifth International Conference on Computational Science and Applications (ICCSA), (2007), pp. 532-538.

[7] X.-c. Yang, J.-p. Li and J.-p. Mou, "An anti-counterfeiting method based on VCS for mobile phone's identification", International Conference on Wavelet Active Media Technology and Information Processing (ICWAMTIP), (2012), pp. 169-172.

[8] S. H. Choi, B. Yang and H. H. Cheung, "RFID tag data processing in manufacturing for track-and-trace anti-counterfeiting", Computers in Industry, vol. 68, (2015), pp. 148-161.

[9] B. Yan and G. Huang, "Application of RFID and Internet of things in monitoring and anti-counterfeiting for products", Business and Information Management (ISBIM), (2008), pp. 392-395.

[10] C.-L. Chen, Y.-Y. Chen and T.-F. Shih, "An RFID authentication and anti-counterfeit transaction Protocol”, Computer, Consumer and Control (IS3C), (2012), pp. 419-422.

[11] M. Radziwon, S. Johansen and H.-G. Rubahn, "Anti-Counterfeit Solution from Organic Semiconductor", Procedia Engineering, vol. 69, (2014), pp. 1405-1409.

[12] Dr. S. D. Thepade and P. Bidwai, "Iris Recognition using Fractional Coefficients of Transforms, Wavelet Transforms and Hybrid Wavelet Transforms, International Conference on Control, Computing, Communication and Materials (ICCCCM),(2013), pp. 1-5.

[13] Abhiram, M. H., Sadhu, C. and Manikantan, K., "Novel DCT based feature extraction for enhanced Iris Recognition", International Conference on Communication, Information \& Computing Technology (ICCICT)], (2012), pp. 1-6.

[14] H.-T. Yin and P. Fu, "Face recognition based on DCT and 2DLDA", in Proc. of the Second International Conference on Innovative Computing, Information and Control, Kumamoto, Japan, (2007), pp. 581-584.

\section{Authors}

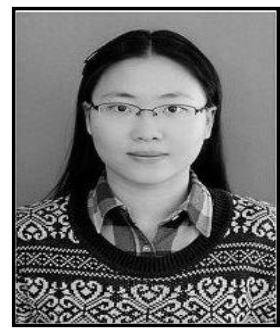

Yue Zhang, was born in JiLin province, China, in 1991. She received the B.S. in Hainan University, Haikou, major in Electronic Information Engineering. Now she is a graduate student at Hainan University.

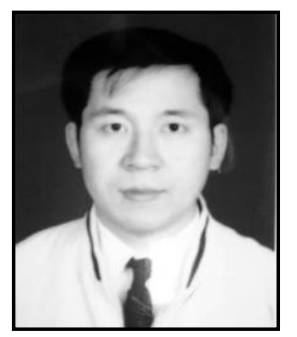

Jingbing Li, was born in 1966. He received the M.S. in Beijing Institute Of Technology, in 1996, and Doctor degree in Chongqing University, in 2007. Now he is a professor in Hainan University. His main research directions are image processing, multimedia information security, digital watermarking, artificial intelligence technology, computer automatic control. 